\title{
Lipid and Biomarker Differences between Raltegravir and Atazanavir/ Ritonavir at 48 Weeks in the Raltegravir Atazanavir Naïve (RAN) Study
}

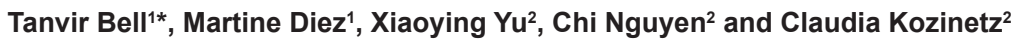

${ }^{1}$ Division of Infectious Diseases, Department of Internal Medicine, University of Texas Health Science Center, Houston, Texas, USA

${ }^{2}$ Department of Pediatrics, Baylor College of Medicine and Study Design and Analysis Core, Baylor-UT Houston Center for AIDS Research, Houston, Texas, USA

\begin{abstract}
Raltegravir and Atazanavir/ritonavir are potent first line therapeutic options for HIV treatment naïve patients. We studied raltegravir (RAL) or atazanavir/ritonavir (ATV/r) both with tenofovir/emtricitabine in a predominantly African American indigent patient population in Houston, Texas. Results from 17 patients at 48 weeks (wk); 9 in the RAL group and 8 in the ATV/r group were available for analyses. We evaluated CD4+ count, CD4\%, HIV RNA viral load (VL) levels, and lipid profile at wk $0,6,14,30$, and 48. IL-6 and homocysteine levels were available at wk 0 and 48 . Among the lipid panel between the 2 groups, triglycerides became statistically significantly lower in the raltegravir group at 14 weeks and persisted at a lower level through $48 \mathrm{wk}$. There were no significant differences in CD4+ cell count, CD4\%, log VL, total cholesterol, LDL, HDL, IL-6, and homocyseine levels. Both RAL and ATV/r in combination with tenofovir/emtricitabine provide similar immunologic and virologic efficacy. There was a slight difference observed in changes in triglyceride levels but other lipid changes were similar. IL-6 and homocystine levels changed to a similar degree with these potent antiretroviral regimens.
\end{abstract}

Keywords: Naive therapy; Lipid levels; Biomarkers; Minority population; Antiretroviral therapy

\section{Introduction}

Raltegravir is the first marketed strand-transfer inhibitor of HIV-1 that was FDA approved in 2007. It is marketed to be used in combination therapy with nucleoside or nucleotide analogue reverse transcriptase inhibitors [N(t)RTIs]. Raltegravir combined with tenofovir and emtricitabine is a preferred regimen to be used in treatment naïve patients with HIV [1]. It has been shown to be a potent regimen for antiretroviral naïve patients [2]. Atazanavir is also a potent antiretroviral drug that has been FDA approved since 2003. Ritonavirboosted atazanavir (ATV/r) in combination with $\mathrm{N}(\mathrm{t}) \mathrm{RTIs}$ is a regimen commonly used in our clinic population. Data in minority indigent populations is lacking for these newer regimens.

\section{Methods}

\section{Study design}

The Raltegravir Atazanavir Naïve (RAN) study (NCT00762892) was a prospective randomized open labeled phase IV trial of antiretroviral treatment-naïve HIV-1-infected individuals. Eligibility criteria included: antiretroviral treatment naïve HIV-1-infected individuals; plan to participate in study for the 96-week study period, followed at Thomas Street Health Center; age greater than 18 years old. Exclusion criteria included:no plan to become pregnant over the study period; not be on a proton pump inhibitor; not undergoing treatment for active tuberculosis; and not having a creatinine clearance by MDRD of $<50$ $\mathrm{ml} / \mathrm{min} / 1.73 \mathrm{~m}^{2}$. Enrollment to this study was from January 2009 to March 2011.

Patients received raltegravir $400 \mathrm{mg}$ twice daily or atazanavir $300 \mathrm{mg}$ per day with ritonavir $100 \mathrm{mg}$ per day both in combination with once daily tenofovir disoproxil fumarate $300 \mathrm{mg} / \mathrm{em}$ tricitabine $200 \mathrm{mg}$ fixed-dose combination (FDC). For simplicity, the groups in subsequent discussion will be referred to the raltegravir group or atazanavir/r group.

The RAN study was performed at Thomas Street Health Center in Houston, TX. This clinic is a large urban clinic in Houston that services

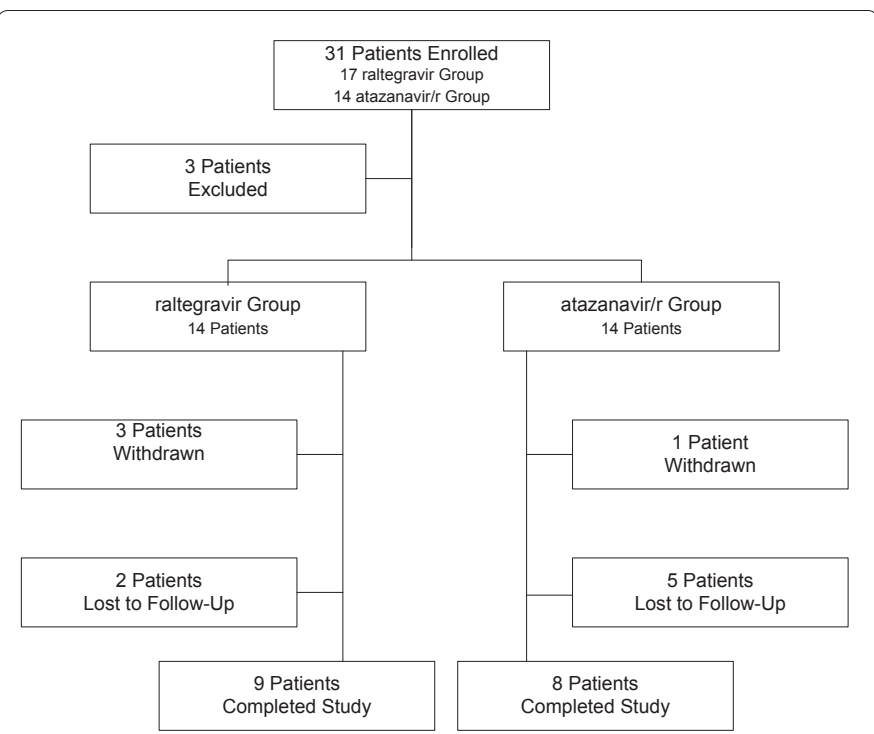

Figure 1: Patients analyzed in RAN.

over 5,500 HIV-1-infected patients that are predominantly indigent (Figure 1).

${ }^{*}$ Corresponding author: Tanvir Bell, Division of Infectious Diseases, Department of Internal Medicine, University of Texas Health Science Center-Houston, 6431 Fannin St., MSB 2.112, Houston, Texas, 77030, USA, Tel: 713-500-6759; Fax: 713-500-5495; E-mail: Tanvir.K.Bell@uth.tmc.edu

Received January 15, 2013; Accepted February 19, 2013; Published February 25, 2013

Citation: Bell T, Diez M, Yu X, Nguyen C, Kozinetz C (2013) Lipid and Biomarker Differences between Raltegravir and Atazanavir/Ritonavir at 48 Weeks in the Raltegravir Atazanavir Naïve (RAN) Study. J AIDS Clin Res 4: 192. doi:10.4172/21556113.1000192

Copyright: (c 2013 Bell T, et al. This is an open-access article distributed under the terms of the Creative Commons Attribution License, which permits unrestricted use, distribution, and reproduction in any medium, provided the original author and source are credited. 
From our original sample of 31 patients, 3 patients were excluded because they did not complete necessary paper work for the Texas AIDS Drug Assistance Program to start medications or did not come back to care after initial visit. Of the remaining 28 patients, 14 were in each arm. We had one patient in the raltegravir arm who stopped taking medications for previously diagnosed Mycobacterium aviumintracellulare and died from multisystem organ failure. She was withdrawn from the study. Two other patients were withdrawn from the study; one was in jail and one became pregnant. In the atazanavir arm, one patient developed jaundice and had atazanavir switched, and he was withdrawn from the study. Two patients in the raltegravir group and 5 patients in the atazanavir group were lost to follow up. We were able to capture data from 17 patients at 48 weeks; 9 in the raltegravir group and 8 in the atazanavir group. We lost more patients after the 48 week time point and thus concluded data collection at 48 weeks.

We evaluated CD4+ cell count, CD4\%, HIV RNA viral load (VL) levels, and lipid profile at wk $0,6,14,30$, and 48 . We evaluated IL-6 levels and homocysteine levels at weeks 0 and 48. Laboratory evaluations were performed at Harris Health System's CLIA certified laboratories. IL-6 and homocysteine were sent out to Quest Diagnostics laboratory.

\section{Statistical analysis}

At baseline, we compared the demographic and behavioral characteristics between patients received raltegravir versus those received atazanavir/r using Chi-square test or Fisher's exact test for categorical variables and two sample t-test for continuous variables.

At each follow up time point, laboratory measurements including CD4, viral load and lipid profile were compared between raltegravir and atazanavir/r groups using Wilcoxon rank sum test as the normality assumptions were violated.

The differences between baseline and the end of 48 weeks for each laboratory measurement were calculated and then compared between groups using Wilcoxon rank sum test.

A p-value $<0.05$ was considered significant for all analyses. All analyses were performed using SAS 9.2 (SAS Institute, Cary, N.C.).

\section{Results}

\section{Patient population}

The study populations were comprised primarily of urban, low socioeconomic status patients (Table 1). The majority were African

\begin{tabular}{|c|l|l|l|}
\hline & $\begin{array}{l}\text { Raltegravir } \\
(\mathbf{n = 1 4 )}\end{array}$ & $\begin{array}{l}\text { Atazanvir/r } \\
(\mathbf{n = 1 4 )}\end{array}$ & p-value \\
\hline Race & & & \\
\hline African American & $8(57 \%)$ & $7(50 \%)$ & $0.06^{*}$ \\
\hline Hispanic & $4(29 \%)$ & $3(21 \%)$ & \\
\hline Caucasian & $2(14 \%)$ & $3(21 \%)$ & \\
\hline Asian & 0 & $1(7 \%)$ & \\
\hline Gender & & & \\
\hline Male & $7(50 \%)$ & $11(78 \%)$ & 0.11 \\
\hline Female & $7(50 \%)$ & $3(21 \%)$ & \\
\hline Drug Screen/Drug Use & & & \\
\hline Positive & $7(50 \%)$ & $4(31 \%)$ & 0.31 \\
\hline Age Mean (SD) & $40.7 \mathrm{yr}(11.1)$ & $38.8 \mathrm{yr}(10.0)$ & 0.64 \\
\hline
\end{tabular}

*Fisher's exact test

Table 1: Baseline demographics.

\begin{tabular}{|l|c|c|c|}
\hline Lab & Raltegravir & Atazanavir/r & p-value \\
\hline CD4 cell count $($ cells/mm $)$ & $136.64(133.87)$ & $156.07(144.92)$ & 0.89 \\
\hline Viral load (copies/mL) & $301,383(283,289)$ & $293,777(311,530)$ & 1.00 \\
\hline Log VL & $5.11(0.76)$ & $5.11(0.67)$ & 1.00 \\
\hline Tot cholesterol $(\mathrm{mg} / \mathrm{dL})$ & $182.77(64.56)$ & $171.79(40.53)$ & 0.92 \\
\hline Triglicerides $(\mathrm{mg} / \mathrm{dL})$ & $132.31(51.50)$ & $140.36(82.10)$ & 0.94 \\
\hline HDL & $45.92(12.45)$ & $41.00(13.21)$ & 0.28 \\
\hline LDL & $110.38(62.45)$ & $102.86(41.40)$ & 0.75 \\
\hline IL6 & $15.11(27.74)$ & $10.65(11.3)$ & 0.60 \\
\hline Homocysteine & $10.36(2.97)$ & $9.69(3.20)$ & 0.44 \\
\hline
\end{tabular}

Table 2: Baseline labs (Mean (SD)) for 28 participants.

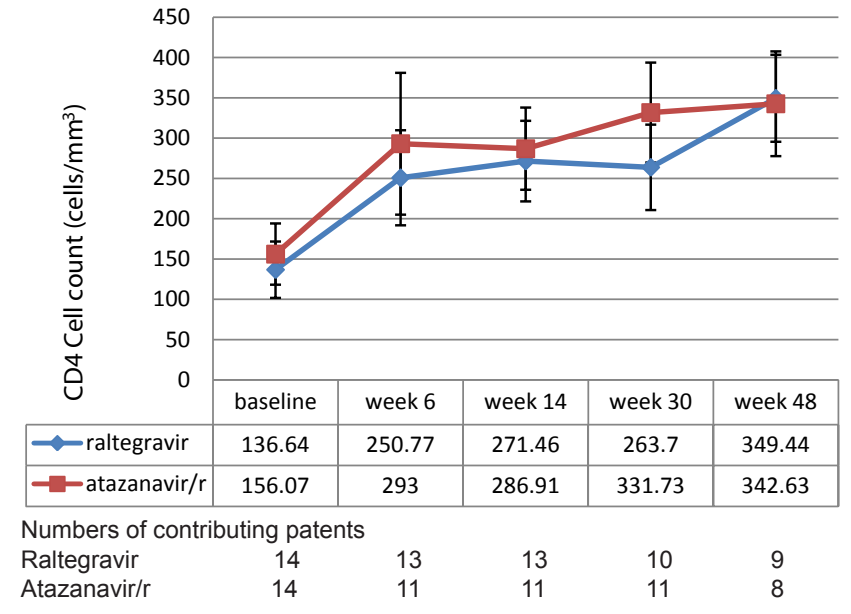

Figure 2: Mean CD4 cell count over time. Error bars represent the standard error of the mean.

American. Many patients also had either a positive drug screen or a history of drug use. More patients with positive drug screen or history of drug screen were in the raltegravir arm than atazanavir/r $50 \%$ vs. $31 \%$, respectively, but not statistically significant $(\mathrm{p}=0.31)$.

At baseline, no differences were found between groups for all variables (Table 2).

Immunologic recovery with an increase in CD4 cell count occurred in both groups. The baseline CD4 cell count was 136.64 cells $/ \mathrm{mm}^{3}$ in the raltegravir arm and 156.07 cells $/ \mathrm{mm}^{3}$ in the atazanavir/r group. The mean CD4 cell count increased in both groups over 48 weeks to 349.44 cells $/ \mathrm{mm}^{3}$ in the raltegravir group and 342.63 cells $/ \mathrm{mm}^{3}$ in the atazanavir/r group (Figure 2).

Virological response was similar in both groups. The HIV RNA viral load decreased from 5.11 log viral load at baseline in both groups to $1.79 \mathrm{log}$ in the raltegravir group and $1.88 \mathrm{log}$ viral load in the atazanavir/r group at week 48 (Figure 3 ).

There were no significant differences in CD4+ cell count and CD4\% change from baseline to week 48 between the two groups.

\section{Lipids}

There were no significant consistent differences in total cholesterol, LDL cholesterol, HDL cholesterol in the two groups but there was a significant difference in triglycerides at week 48 . At week 30 , the atazanavir/r group had increased total cholesterol and LDL-c when compared to the raltegravir group but this statistically significant 
increase did not persist at 48 weeks. At week 6, triglycerides were found to have marginal significant difference between the groups with a decrease in mean levels in the raltegravir group ( $\mathrm{p}$-value $=0.0557$ ). Over the follow-up period from week 14 to week 48, the triglyceride difference remained consistent and significant $(p<0.05$, see Figure 4$)$. At week 6, of the 11 patients who had labs drawn in the atazanavir/r group, lipids were not performed in 4 patients due to labs not being done either because of patients not fasting or due to a missed order. The mean values of triglycerides at week 48 were $105 \pm 39.68$ in the raltegravir group vs. $179 \pm 56.72$ in the atazanavir/r group $(\mathrm{p}=0.01)$.

Treatment for hyperlipidemia was rarely begun in this population in the 48 weeks studied. One patient in the raltegravir arm had atorvastatin begun at week 30 . One patient in the atazanavir/ $\mathrm{r}$ arm had fenofibrate begun at week 14 .

\section{Demographic and laboratory differences in patients who complete week 48}

Discontinuation rate did not affect the demographic makeup of the group. Among the patients that were able to complete 48 weeks,
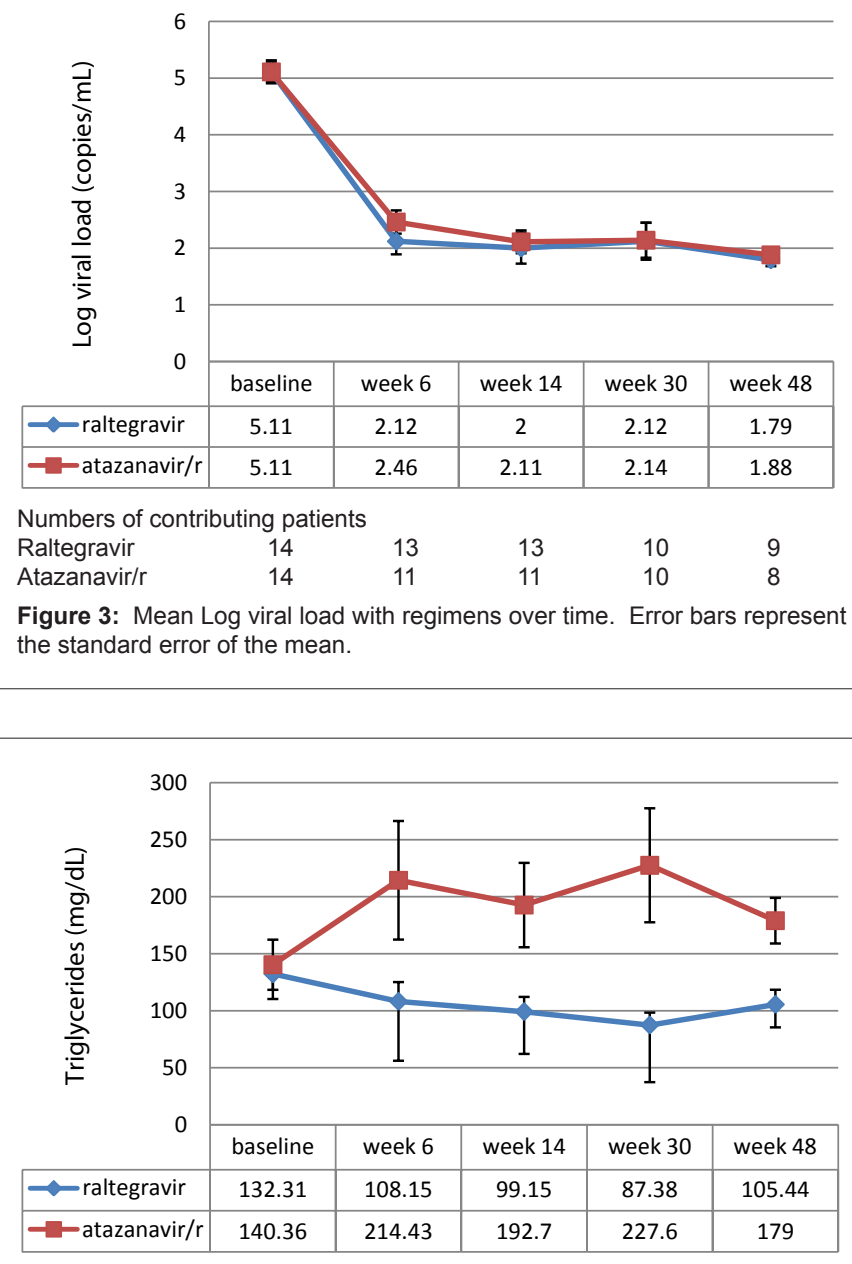

Numbers of contributing patients

$\begin{array}{llllcl}\text { raltegravir } & 14 & 13 & 13 & 8 & 9 \\ \text { atazanavir/r } & 14 & 7 & 10 & 10 & 8\end{array}$

Figure 4: Mean triglycerides change from baseline to 48 weeks $(p<0.05$ from week 14 to week 48).Error bars represent the standard error of the mean.

\begin{tabular}{|c|l|l|l|}
\hline & Raltegravir (n=9) & Atazanvir/r (n=8) & p-value \\
\hline Race & & & \\
\hline African American & $5(56 \%)$ & $5(63 \%)$ & $0.77^{*}$ \\
\hline Hispanic & $3(33 \%)$ & $1(13 \%)$ & \\
\hline Caucasian & $1(11 \%)$ & $1(25 \%)$ & \\
\hline Asian & $0(0 \%)$ & $1(13 \%)$ & \\
\hline Gender & & & $1.00^{*}$ \\
\hline Male & $6(67 \%)$ & $6(75 \%)$ & \\
\hline Female & $3(33 \%)$ & $2(25 \%)$ & \\
\hline $\begin{array}{l}\text { Drug Screen/ } \\
\text { Drug Use }\end{array}$ & & & $0.33^{*}$ \\
\hline Positive & $5(56 \%)$ & $2(25 \%)$ & 0.85 \\
\hline Age (yr) & $42.8(13.2)$ & $43.8(8.5)$ & \\
\hline
\end{tabular}

* Fisher's exact test

Table 3: Demographics of those who completed week 48 visit.

the racial and gender make of the participants remained similar from the baseline population. It was still predominantly African American with a mean age of about 43 years. As in the total population, there were more patients in the raltegravir arm with a positive drug screen or a history of drug use (Table 3). We compared the demographic and laboratory characteristics among those who were retained in the study and those that were lost to follow up (Table 4).

We also checked the baseline differences between patients remaining in the cohort at week 48 and those lost to follow up. No significant difference was detected.

Comparing changes in mean values between baseline and week 48 were not significantly different among the two groups (Table 5 ). The changes were calculated among 17 patients remaining in the study at week 48 .

\section{Biomarkers}

IL-6 and homocysteine were measured as markers of immune inflammation. IL- 6 decreased in both groups in these potent regimens the mean value of IL6 decreased 2.71 with a standard deviation of 1.63 in the raltegravir arm. In the atazanvir/ $\mathrm{r}$ arm the mean value of IL- 6 decreased 4.47 with a standard deviation of $2.51(\mathrm{p}=0.73)$.

\section{Discussion}

Integrase inhibitors are a newer class of antiretroviral drugs for the treatment of HIV-1-infected individuals. Initial evaluation of use of raltegravir in antiretroviral-treatment naïve patients was performed in Protocol 004 and 021trial. Protocol 004 compared the established dose of raltegravir $400 \mathrm{mg}$ daily to efaverinz $600 \mathrm{mg}$ daily in combination with tenofovir disoproxil fumarate/emtricitabine FDC. At 96 weeks, the virological success and immunologic response among 198 HIV-1infected individuals were similar in the two groups [3]. In Protocol 021, the study also known as the STARTMRK, 566 patients were randomly assigned to receive either raltegravir or efaverinz in combination with tenofovir disoproxil fumarate/emtricitabine FDC [4]. This study demonstrated that raltegravir was non-inferior to efaverinz at 48 weeks. In this study more patients achieved viral load at less than 50 copies $/ \mathrm{mL}$ at wk 2-16. A greater increase in mean CD4 cell count occurred in the raltegravir arm.

A randomized clinical trial comparing raltegravir to ritonavirboosted atazanavir has not been reported thus far. Both options 
combined with tenofovir disoproxil fumarate/emtricitabine FDC are preferred regimens by the DHHS [1] and IAS guidelines [5]. In our study, which compared a ritoanvir-boosted protease inhibitor rather than the non-nucleoside reverse transcriptase inhibitor, the viralogic decline was similar to the integrase inhibitor. CD4 cell count increase at 48 weeks was similar. In our patient population, among those who remained on study, raltegravir and ritonavir-boosted atazanavir provided similar immunologic and viralogic efficacy.

In the continuation of Protocol 004 at 96 weeks, raltegravir was found to have minimal impact on total or LDL cholesterol, lowdensity lipoprotein cholesterol and triglycerides [3]. At week 48, the STARTMRK show a small increases in total cholesterol, LDLcholesterol and HDL levels and slight decreased in triglyceride levels among subject trated with raltegravir. In the efaverinz arm, the changes were significantly increased from baseline with triglicerides increasing significantly compared with raltegravir [4] as we found in our study.

The hyperlipidemia may result in increased risk of cardiovascular morbidity and mortality. Many cohorts have found that HIV-1infected patients have higher cardiovascular events [6,7]. HIV infected patients in a Massachusetts cohort had more dyslipidemia than HIV uninfected cohort. Increased exposure to protease inhibitors in the D: A: D cohort was correlated with increased risk of myocardial infarction. This was in part due to dyslipidemia [8]. Atazanavir/r often produces less increase in cholesterol than other protease inhibitors. A metaanalysis of randomized control trials comparing ritonavir-boosted atazanavir with other ritonavir-boosted protease inhibitors found

\begin{tabular}{|l|c|c|c|}
\hline & $\begin{array}{c}\text { Patients } \\
\text { remained in the } \\
\text { cohort (n=17) }\end{array}$ & $\begin{array}{c}\text { Patients lost to } \\
\text { follow up } \\
\text { (n=11) }\end{array}$ & p-value \\
\hline Demographic characteristics & & & \\
\hline Race & $10(59 \%)$ & $5(46 \%)$ & 0.80 \\
\hline African American & $4(23 \%)$ & $3(27 \%)$ & \\
\hline Hispanic & $2(12 \%)$ & $3(27 \%)$ & \\
\hline Caucasian & $1(6 \%)$ & $0(0 \%)$ & \\
\hline Asian & & & \\
\hline Gender & $12(71 \%)$ & $6(55 \%)$ & 0.44 \\
\hline Male & $5(29 \%)$ & $5(45 \%)$ & \\
\hline Female & & & \\
\hline Drug Screen/Drug Use & $7(41 \%)$ & $4(40 \%)$ & 1.00 \\
\hline Positive & & & \\
\hline Baseline laboratory tests & $147.2(134.9)$ & $145(147.5)$ & 0.97 \\
\hline CD4 (cells/uL) & $10.29(8.46)$ & $10.55(8.35)$ & 0.94 \\
\hline CD4 percent & $5.00(0.70)$ & $5.28(0.70)$ & 0.30 \\
\hline Log VL (copies/ml) & $179.7(51.04)$ & $173.3(57.31)$ & 0.76 \\
\hline Total Cholesterol (mg/dL) & $138.3(77.11)$ & $133.9(55.3)$ & 0.87 \\
\hline Triglycerides (mg/dL) & $46.56(11.85)$ & $38.73(13.36)$ & 0.12 \\
\hline HDL (mg/dL) & $105.6(53.08)$ & $107.8(52.12)$ & 0.91 \\
\hline LDL (mg/dL) & $7.27(9.45)$ & $21.55(30.04)$ & 0.15 \\
\hline IL-6 & $9.94(2.89)$ & $10.17(3.42)$ & 0.84 \\
\hline Homocysteine (units) & & \\
\hline Changes & & & \\
\hline
\end{tabular}

Changes in laboratory values between baseline and week 48

Table 4: Demographics and laboratory values for those that remained and those that were lost to follow up.

\begin{tabular}{|l|c|c|c|}
\hline Lab & Raltegravir $(\mathrm{n}=9)$ & Atazanavir/r $(\mathrm{n}=8)$ & $\mathrm{p}$-value \\
\hline CD4 change(cells/ $\mathrm{\mu L})$ & $192(26)$ & $205(27)$ & 0.81 \\
\hline Log VL (copies/ml) & $-3.05(0.28)$ & $-3.29(0.26)$ & 0.67 \\
\hline Total Cholesterol $(\mathrm{mg} / \mathrm{dL})$ & $-0.25(7.66)$ & $8.13(11.96)$ & 0.52 \\
\hline Triglycerides $(\mathrm{mg} / \mathrm{dL})$ & $-15.50(18.47)$ & $16.88(40.89)$ & 0.52 \\
\hline HDL $(\mathrm{mg} / \mathrm{dL})$ & $-1.5(2.58)$ & $-1.38(3.08)$ & 0.90 \\
\hline LDL $(\mathrm{mg} / \mathrm{dL})$ & $4.13(7.54)$ & $5.88(14.46)$ & 0.88 \\
\hline IL6 & $-2.71(1.63)$ & $-4.47(2.51)$ & 0.73 \\
\hline Homocysteine (units) & $0.53(0.83)$ & $0.10(0.94)$ & 0.73 \\
\hline
\end{tabular}

Table 5: Change in immunologic, virologic, lipid and biomarker values (mean (SD)) between week 48 and baseline.

that lipid concentrations of total cholesterol, LDL cholesterol, and triglycerides were lower in the boosted atazanavir armat 48 weeks [9]. Protocol 004 found no clinically relevant changes in cholesterol when comparing raltegravir to efaverinz [3]. At 48 weeks, the mean changes in total cholesterol, LDL-cholesterol, HDL cholesterol and triglyceride concentrations in STARTMRK trial were smaller for raltegravir vs. efaverinz. There was a small decline in triglycerides noted in the raltegravir arm [4].

Several biomarkers are being increasingly evaluated as potential surrogate markers of immune inflammatory and atherosclerosis risk. Higher levels of high sensitivity C-Reactive Protein (hsCRP), D-dimer and IL- 6 at study entry correlated with all-cause mortality in the SMART study [10]. IL-6 and homocysteine effect of antiretroviral drugs on biomarkers of inflammation is an emerging area of study for HIV medications. IL-6 is an important modulator of inflammation and atherosclerosis. Elevated levels of homocysteine are associated with increased atherosclerosis. We did not find any significant changes in IL-6 and homocysteine between the two arms of this study but the atazanavir/r group had greater mean decrease IL-6 levels than in the raltegravir group ( -4.47 vs. -2.71 , respectively), though this was not statistically significant. Patients that were lost to follow up had higher levels of IL- 6 compared with those that stayed in the study though this was not statistically significant. There was higher variability in the IL-6 levels at baseline among those that stayed.

Our study evaluated a unique predominantly minority population. This population is classically under represented in clinical studies. The number of patients lost to follow up is a limitation of our study and lead to us terminate the city at 48 weeks. This was a challenging urban population that were not the best typical clinical study candidates. Recent data have revealed that many patients are not retained in care and remain undetectable during current times [11]. Many clinical studies do have increased retention for clinical trials but minorities are also often under represented. In our trial they are well represented. Drug use is often excluded in clinical trials. A large proportion had a positive drug screen or used drugs. More patients who used drugs were in the raltegravir group completing 48 weeks yet this did not impact results. An equal proportion of patients using drugs stayed in the trial and were lost to follow up of the trail at around $40 \%$.

Limitations to consider in this study are the relatively small sample size and loss to follow-up. However, the unique characteristics of the study population make important contributions to the treatment for HIV. Medical records of patients that were lost to follow up was reviewed, the county indigent hospital records were reviewed to ensure that no serious adverse effects occurred. We also attempted multiple times to contact patients who were lost to follow up. A minority 
Citation: Bell T, Diez M, Yu X, Nguyen C, Kozinetz C (2013) Lipid and Biomarker Differences between Raltegravir and Atazanavir/Ritonavir at 48 Weeks in the Raltegravir Atazanavir Naïve (RAN) Study. J AIDS Clin Res 4: 192. doi:10.4172/2155-6113.1000192

population from an urban clinic with a high incidence of drug abuse is an unusual and difficult population to enroll and follow in a clinical trial. It is important, however, to make efforts to include such populations in studies as the outcomes will be more relevant to the sub-populations affected by HIV and to public health in general.

Larger randomized studies that compare raltegravir with atazanavir/ritonavir in combination with tenofovir disoproxil fumarate/emtricitabine FDC needs to be conducted. One trial within the Adult AIDS Clinical Trials Group (ACTG 5257) has these two regimen options as two of the three arms as options for treating naïve individuals. Analysis of the trial is underway.

\section{Acknowledgements}

This work was supported by Merck and Co. Investigator-Initiated Studies Program (to Tanvir Bell).

Statistical analysis was provided by the Design and Analysis Core of the Baylor-UTHoustonCenter for AIDS Research.

T. K. Bell served as the principle investigator of the trial. She designed the study and facilitated multiple aspects of the study. T.K. Bell serves as a consultant to Merck.

M. Diez served as study coordinator and assisted in the day to day needs of the study

X. Yu, C. Nguyen, and C. Kozinetz contributed to data analysis

Roberto Arduino, MD offered mentorship and guidance for this project.

\section{References}

1. Panel on Antiretroviral Guidelines for Adults and Adolescents (2013) Guidelines for the use of antiretroviral agents in HIV-1-infected adults and adolescents. Department of Health and Human Services.

2. Markowitz M, Nguyen BY, Gotuzzo E, Mendo F, Ratanasuwan W, et al. (2007) Rapid and durable antiretroviral effect of the HIV-1 Integrase inhibitor raltegravir as part of combination therapy in treatment-naive patients with HIV-1 infection: results of a 48-week controlled study. J Acquir Immune Defic Syndr 46: 125133.

3. Markowitz M, Nguyen BY, Gotuzzo E, Mendo F, Ratanasuwan W, et al. (2009) Sustained antiretroviral effect of raltegravir after 96 weeks of combination therapy in treatment-naive patients with HIV-1 infection. J Acquir Immune Defic Syndr 52: 350-356.

4. Lennox JL, DeJesus E, Lazzarin A, Pollard RB, Madruga JV, et al. (2009) Safety and efficacy of raltegravir-based versus efavirenz-based combination therapy in treatment-naive patients with HIV-1 infection: a multicentre, doubleblind randomised controlled trial. Lancet 374: 796-806.

5. Thompson MA, Aberg JA, Hoy JF, Telenti A, Benson C, et al. (2012) Antiretroviral treatment of adult HIV infection: 2012 recommendations of the International Antiviral Society-USA panel. JAMA 308: 387-402.

6. Currier JS, Taylor A, Boyd F, Dezii CM, Kawabata H, et al. (2003) Coronary heart disease in HIV-infected individuals. J Acquir Immune Defic Syndr 33: 506512.

7. Triant VA, Lee H, Hadigan C, Grinspoon SK (2007) Increased acute myocardial infarction rates and cardiovascular risk factors among patients with human immunodeficiency virus disease. J Clin Endocrinol Metab 92: 2506-2512.

8. DAD Study Group, Friis-Møller N, Reiss P, Sabin CA, Weber R, et al. (2007) Class of antiretroviral drugs and the risk of myocardial infarction. N Engl J Med 356: $1723-1735$.

9. Carey D, Amin J, Boyd M, Petoumenos K, Emery S (2010) Lipid profiles in HIVinfected adults receiving atazanavir and atazanavir/ritonavir: systematic review and meta-analysis of randomized controlled trials. J Antimicrob Chemother 65 1878-1888.

10. Kuller LH, Tracy R, Belloso W, De Wit S, Drummond F, et al. (2008) Inflammatory and coagulation biomarkers and mortality in patients with HIV infection. PLoS Med 5: e203.

11. Gardner EM, McLees MP, Steiner JF, Del Rio C, Burman WJ (2011) The spectrum of engagement in HIV care and its relevance to test-and-treat strategies for prevention of HIV infection. Clin Infect Dis 52: 793-800. 